\title{
Skin Autofluorescence as a Predictor of First Heart Failure Hospitalization in Patients With Heart Failure With Preserved Ejection Fraction
}

\author{
Takashi Hitsumoto
}

\begin{abstract}
Background: An autofluorescence (AF) reader can be used to diagnose skin AF non-invasively by measuring local accumulation of advanced glycation end-products. A number of studies have investigated the relationships between skin AF and cardiovascular disease. However, data regarding the usefulness of skin $\mathrm{AF}$ as a predictor of chronic heart failure remain limited. This prospective study aimed to elucidate the usefulness of skin AF as a predictor of first heart failure (HF) hospitalization in patients with HF with preserved ejection fraction (HFpEF).
\end{abstract}

Methods: A total of 412 outpatients with HFpEF with no history of HF hospitalization were enrolled. Patients were assigned to either the low (group L; skin $\mathrm{AF} \leq 2.9$ arbitrary units $(\mathrm{AU}) ; \mathrm{n}=303$ ) or the high (group $\mathrm{H}$; skin $\mathrm{AF} \geq 3.0 \mathrm{AU} ; \mathrm{n}=109$ ) group according to optimal skin AF cut-off levels determined using receiver operating characteristic curves. Clinical parameters and the usefulness of skin AF as a predictor of first HF hospitalization were evaluated.

Results: The E/e' ratio as a marker of left ventricular diastolic function was significantly higher in group $\mathrm{H}$ patients than in group $\mathrm{L}$ patients at baseline (group H, $11.8 \pm 3.8$; group L, $10.6 \pm 3.3 ; \mathrm{P}=0.002$ ). During the 72.7-month follow-up period, $43 \mathrm{HF}$ cases were hospitalized (group L, 15 cases; group H, 28 cases; $\mathrm{P}<0.001$, log-rank test). Multivariate Cox regression analyses revealed that group $\mathrm{H}$ exhibited a significantly higher risk of first HF hospitalization than did group L (hazard ratio, 2.26; 95\% confidence interval, $1.21-3.52 ; \mathrm{P}=0.014$ ).

Conclusions: The present study demonstrated that skin AF can predict the risk of first HF hospitalization in patients with HFpEF. Prospective studies, including intervention therapies, are required to validate our observations.

Keywords: Skin autofluorescence; Heart failure with preserved ejection fraction; First heart failure hospitalization; Left ventricular diastolic function; Cardio-ankle vascular index; Inflammation

Manuscript submitted May 9, 2020, accepted May 19, 2020

Published online June 3, 2020

Hitsumoto Medical Clinic, 2-7-7, Takezakicyou, Shimonoseki City, Yamaguchi 750-0025, Japan. Email: thitsu@jcom.home.ne.jp

doi: https://doi.org/10.14740/cr1097

\section{Introduction}

In recent years, extended life expectancy and other factors have led to an increase in the incidence of chronic heart failure $(\mathrm{CHF})$ worldwide $[1,2]$. Indeed, numerous patients with $\mathrm{CHF}$, including those in asymptomatic phases, are encountered in daily clinical practice. Moreover, patients with CHF and a history of heart failure (HF) hospitalization reportedly have a poor prognosis because of re-hospitalization due to HF, cardiovascular diseases, or other adverse events [3, 4]. Thus, prompt diagnosis and therapy for patients with $\mathrm{CHF}$ are critical to reducing the incidence of HF hospitalization.

In the clinical setting, CHF can be divided into two or three types by left ventricular ejection fraction. In particular, recent studies have investigated patients with HF with preserved ejection fraction (HFpEF). Contrary to the remarkable advances in the development of therapeutic agents with proven benefit in HF with reduced ejection fraction (HFrEF), evidence-based therapeutic options lack for patients with HFpEF $[5,6]$. Moreover, similar to HFrEF, HFpEF also has a poor prognosis $[5,7]$. Thus, it is imperative to investigate novel diagnostic and therapeutic options for HFpEF.

Advanced glycation end-products (AGEs) play an important role in the pathophysiology of cardiovascular disease. Among the methods used to evaluate AGEs, skin autofluorescence (AF) is known to be a simple and reliable marker in vivo, and recent clinical studies have indicated that skin AF levels are significantly associated with cardiovascular disease incidence and risk factors [8-10]. Moreover, several studies have found an association between skin $\mathrm{AF}$ and the pathogenesis of HF [11-13]. However, data on the usefulness of skin AF as a predictor of CHF are limited. Thus, this prospective study aimed to elucidate the usefulness of skin $\mathrm{AF}$ as a predictor of first HF hospitalization in patients with HFpEF.

\section{Materials and Methods}

\section{Patients}

Between August 2011 and July 2013, 436 patients with HFpEF (left ventricular ejection fraction as estimated by echocardiography $>50 \%$ ) and no history of HF hospitalization 


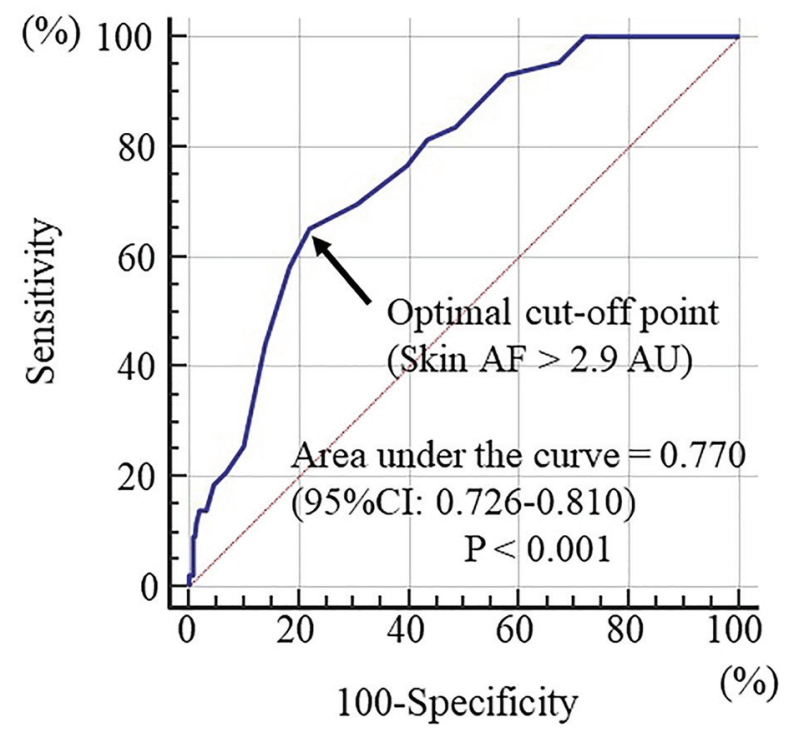

Figure 1. Prediction of first heart failure hospitalization at follow-up period using skin autofluorescence. Receiver operating characteristics to determine the optimal cut-off point of skin AF for the first heart failure hospitalization. The area under curve was $0.770(P<0.001)$. Maximum Youden index indicated that a skin AF > 2.9 arbitrary units was optimal cut-off point to predict the first heart failure hospitalization, indicating a sensitivity of $65.1 \%$ and a specificity of $78.1 \%$. Arrow indicates the optimal cut-off point. AF: autofluorescence; AU: arbitrary units; $\mathrm{Cl}$ : confidence interval.

presented to the Hitsumoto Medical Clinic, Yamaguchi, Japan. Of these, 24 patients with lack of baseline clinical data were excluded. The remaining 412 patients were prospectively included in this study. CHF was defined according to the ACC/AHA 2005 Guidelines for the Diagnosis and Management of Heart Failure in Adults [14], and patients with stage $\mathrm{B}$ (patients who are asymptomatic but demonstrate structural heart diseases such as left ventricular hypertrophy, ischemic heart disease and valvular heart disease) and stage $\mathrm{C}$ (patients with HF symptoms) were enrolled in this study. The enrolled patients included $105(25.5 \%)$ males and $307(74.5 \%)$ females (mean age, $73 \pm 8$ years). Patients were assigned to either the low group (group L; skin $\mathrm{AF} \leq 2.9$ arbitrary units $(\mathrm{AU}) ; \mathrm{n}=303$ ) or the high group (group $\mathrm{H}$; skin $\mathrm{AF} \geq 3.0$ AU; $n=109$ ) according to the optimal skin AF cut-off levels determined using receiver operating characteristic curves (Fig. 1). The study protocol was approved by the Local Ethics Committee of the Hitsumoto Medical Clinic (approval number: HMC-2011-6).

\section{Skin AF measurement}

Skin AF was measured using a commercial device (AGE Reader ${ }^{\mathrm{TM}}$; DiagnOptics, Groningen, the Netherlands), as previously described $[15,16]$. AF was defined as the average light intensity per nanometer in the spectrum between 300 and 420 $\mathrm{nm}$. Skin AF levels were expressed in AU. All measurements were performed at the volar side of the lower arm, approxi- mately $10-15 \mathrm{~cm}$ below the elbow, while the patients were in a sitting position. The value of pentosidine, a major AGEs component, was measured by skin biopsy taken at the volar side of the lower arm and has been found to correlate well with skin AF [17]. The validity and reliability of measuring skin AF levels in the Japanese population by this method were established previously [16].

\section{Assessment of clinical parameters}

The present study evaluated various clinical parameters, including classical risk factors for cardiovascular disease, history of ischemic heart disease, echocardiographic findings, hemoglobin levels, kidney function, brain natriuretic peptide (BNP) levels, high-sensitivity C-reactive protein (hs-CRP) levels as a marker of inflammation and cardio-ankle vascular index (CAVI) as a marker of arterial function. Obesity was identified using body mass index (BMI) data, with BMI calculated as the body weight $(\mathrm{kg})$ divided by the squared body height $\left(\mathrm{m}^{2}\right)$. Smoking was defined as smoking at least one cigarette per day for the past 28 days. A history of ischemic heart disease was defined as having previous myocardial infarction and/or significant angiography-proven stenosis. Hypertension was defined as a systolic blood pressure $\geq 140 \mathrm{~mm} \mathrm{Hg}$, diastolic blood pressure $\geq 90 \mathrm{~mm} \mathrm{Hg}$ and/or administration of antihypertensive medications. Dyslipidemia was defined as low-density lipoprotein cholesterol levels $\geq 140 \mathrm{mg} / \mathrm{dL}$, highdensity lipoprotein cholesterol levels $\leq 40 \mathrm{mg} / \mathrm{dL}$, triglyceride levels $\geq 150 \mathrm{mg} / \mathrm{dL}$ and/or ongoing treatment for dyslipidemia. Diabetes mellitus was defined as having fasting blood glucose levels $\geq 126 \mathrm{mg} / \mathrm{dL}$ or hemoglobin A1c levels $\geq 6.5 \%$, as stipulated by the National Glycohemoglobin Standardization Program, and/or taking antidiabetic treatment. Echocardiography was performed using a commercial device (HI VISION Avius, Hitachi Medical Corporation, Tokyo, Japan). Valvular heart disease comprised aortic or mitral valve disease (aortic stenosis, aortic regurgitation, mitral stenosis, or mitral regurgitation). Moreover, left ventricular wall thickness, extended period diameter, ejection fraction, left atrial dimension and E/e' ratio as a marker of left ventricular diastolic function were evaluated using echocardiography. Estimated glomerular filtration rate (eGFR) was calculated using the adjusted Modification of Diet in Renal Disease equation, as proposed by the Working Group of the Japanese Chronic Kidney Disease Initiative [18]. BNP levels were measured using a commercial kit (SHIONOSPOT Reader; Shionogi \& Co., Osaka, Japan). CAVI was measured using VaSera VS-1000 (Fukuda Denshi Co. Ltd, Tokyo, Japan) according to a previously described method [19]. Briefly, the brachial and ankle pulse waves were determined using inflatable cuffs with the pressure maintained between 30 and $50 \mathrm{~mm} \mathrm{Hg}$ to ensure that the cuff pressure had minimal effects on systemic hemodynamics. Following a 10-min rest in a quiet room, blood and pulse pressures were simultaneously measured with the participant in the supine position. Mean values for the left and right sides were used for the statistical evaluation of the CAVI. The CAVI was calculated using the following formula derived from the Bramwell-Hill equation: $\mathrm{CAVI}=\mathrm{a} \times((2 \rho / \Delta \mathrm{P}) \times \ln (\mathrm{Ps} / \mathrm{Pd}) \times$ 
$\left.\mathrm{PWV}^{2}\right)+\mathrm{b}$, where $\mathrm{a}$ and $\mathrm{b}$ are constants, $\rho$ is blood density, $\Delta \mathrm{P}$ is Ps - Pd, Ps is systolic blood pressure, $\mathrm{Pd}$ is diastolic blood pressure and PWV is pulse wave velocity. The CAVI may be less accurate in patients with a non-sinus rhythm or obstructive arteriosclerosis; therefore, patients with chronic atrial fibrillation and/or obstructive arteriosclerosis (ankle-brachial index $<0.9$ ) were excluded. Reportedly, the average variation within the CAVI is $<5 \%$, which is sufficiently small for clinical use, indicating good reproducibility [19].

\section{Patient follow-up}

The follow-up was terminated in December 2019. Patients were followed up for a median of 72.7 months (range, $6-100$ months). The endpoint of this study was the incidence of HF hospitalization.

\section{Statistical analysis}

Data were analyzed using MedCalc for Windows (version 14.8.1; MedCalc Software, Ostend, Belgium) and StatView J5.0 (HULINKS, Tokyo, Japan). Receiver operating characteristic curves were constructed, and the maximum Youden index [20] was used to determine the optimal skin AF cut-off levels for predicting HF hospitalization. Data are presented as means and standard deviations. Between-group comparisons were performed using Student's $t$-test, the Mann-Whitney U-test, or the Chi-squared test. Event-free survival rate curves were plotted using Kaplan-Meier analysis, and differences between the curves were evaluated using the log-rank test. Multivariate analysis was performed using Cox regression. Probability (P) values of $<0.05$ were considered to indicate statistical significance.

\section{Results}

\section{Patient characteristics}

The characteristics of all patients at registration are summarized in Table 1. The mean skin AF for groups L and $\mathrm{H}$ was 2.4 and 3.3 AU, respectively. The following factors were significantly higher in group $\mathrm{H}$ than in group L: incidence of stage $\mathrm{C}$, presence of diabetes mellitus, E/e' ratio, BNP levels, hs-CRP levels and CAVI. However, eGFR was significantly lower in group $\mathrm{H}$ than in group $\mathrm{L}$.

\section{Kaplan-Meier curve analysis}

The Kaplan-Meier curve for the incidence of HF hospitalization is displayed in Figure 2. During the follow-up period, 43 HF cases were hospitalized (group L, 15 cases; group H, 28 cases). The Kaplan-Meier curve demonstrated that group $\mathrm{H}$ exhibited a significantly higher incidence of HF hospitalization than did group $\mathrm{L}(\mathrm{P}<0.001, \log$-rank test $)$.

\section{Clinical parameters at registration of patients with and without HF hospitalization}

The clinical parameters at registration of all patients with and without HF hospitalization are summarized in Table 2. Age, incidence of stage $\mathrm{C}$, smoking status, presence of diabetes mellitus, E/e' ratio, BNP levels, hs-CRP levels and CAVI were considerably higher, whereas eGFR and $\beta$-blocker use were considerably lower in patients with HF hospitalization than in patients not experiencing HF hospitalization.

\section{Multivariate analysis}

The results of multivariate Cox regression analysis of the incidence of HF hospitalization are summarized in Table 3. Eleven variables were included, which were all identified as significant factors for HF hospitalization in the univariate analysis. Among these, six variables (stage $\mathrm{C}$, CAVI, group $\mathrm{H}$, diabetes mellitus, hs-CRP levels and E/e' ratio) were associated with a considerable risk of HF hospitalization (Table 3, all patients). However, five variables (CAVI, group H, hs-CRP levels, diabetes mellitus and E/e' ratio) were associated with a considerable risk of HF hospitalization in patients with stage $\mathrm{C}$ (Table 3 , stage $\mathrm{C}$ patients).

\section{Discussion}

Previous studies have reported an association between HF symptoms, diabetes mellitus and prognosis of CHF [21-23]. Similarly, this study demonstrated that these factors were predictors of first HF hospitalization in patients with HFpEF. Moreover, this study demonstrated that high skin AF $(\geq 3.0$ $\mathrm{AU})$ was an important predictor of first HF hospitalization in patients with HFpEF. However, the E/e' ratio as a marker of left ventricular diastolic function, CAVI as a novel marker of arterial function and hs-CRP levels as an inflammation marker were also identified as predictors of first HF hospitalization.

Left ventricular diastolic function is considered one of the most important factors of HF prognosis in HFpEF patients [24]. The results of this study also revealed the E/e' ratio as a predictor of first HF hospitalization in patients with HFpEF. However, AGEs/AGE receptors (RAGEs) may affect left ventricular diastolic function through several pathways [25, 26]. Moreover, several clinical reports exist demonstrating a significant relationship of left ventricular diastolic function and skin AF $[27,28]$. The results of this study also indicate that a high skin AF level reflects left ventricular diastolic dysfunction. Therefore, the reason why patients with high skin AF are at increased risk of HF hospitalization might be explained by left ventricular dysfunction caused by AGEs/RAGEs.

A couple of clinical studies have indicated the importance of myocardial injury in the prognosis of $\mathrm{CHF}$, including HF hospitalization [29, 30]. Moreover, basic research studies have reported several pathways in which AGEs/RAGEs could be associated with myocardial injury, with evidence of 
Table 1. Characteristics of Patients

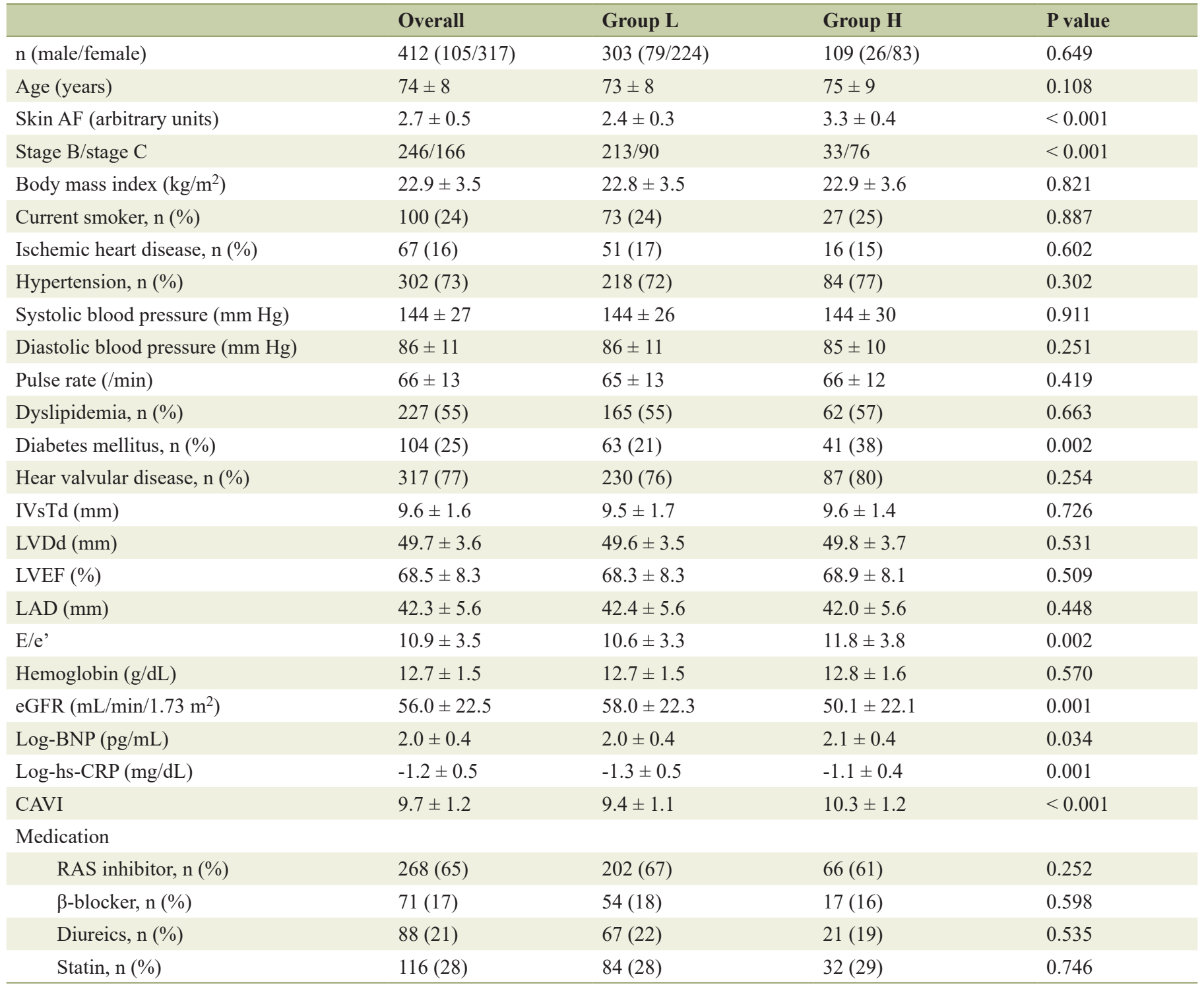

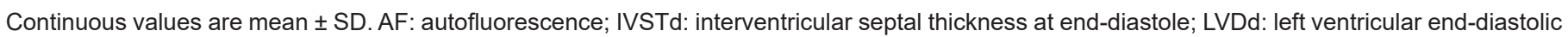

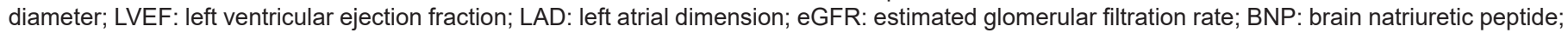
hs-CRP: high-sensitivity C-reactive protein; CAVI: cardio-ankle vascular index; RAS: renin-angiotensin system.

significant increase in AGEs levels in the heart, particularly in the cardiomyocytes [31]. This research also indicated that AGEs-induced cardiomyocyte dysfunction might be linked to mitochondrial membrane depolarization and reduced GSK-3 $\beta$ inactivation, which are events that can be prevented by RNA interference knockdown of RAGEs expression. However, others have found that RAGEs affected ischemia/reperfusion injury in the myocardium [32]. Hofmann et al reported on the relationship between AGEs-modified cardiac tissue collagen levels and skin AF and found a significant relationship between cardiac tissue glycation and skin AF [33]. Moreover, they also clarified that the AGEs found at the volar side of the lower arm appear to reflect the level of AGEs/RAGEs in the cardiomyocytes. In fact, there are several clinical studies that show significant relationships between skin AF and biomarkers of myocardial injury $[13,34]$. Although markers of myocardial injury were not evaluated in the present study, previous studies have shown that AGEs/RAGEs are believed to play important roles in the progression of myocardial injury; consequently, HFpEF patients with high skin AF may be particularly prone to becoming hospitalized due to HF.

The CAVI is known as a proxy for systemic arterial stiffness which is independent of blood pressure levels [19]. Moreover, there is evidence that the CAVI also reflects endothelial function $[35,36]$. Thus, previous studies have clarified that the CAVI is a useful physiological marker to evaluate arterial function in vivo. However, several clinical studies have reported an association between arterial dysfunction, including increased 


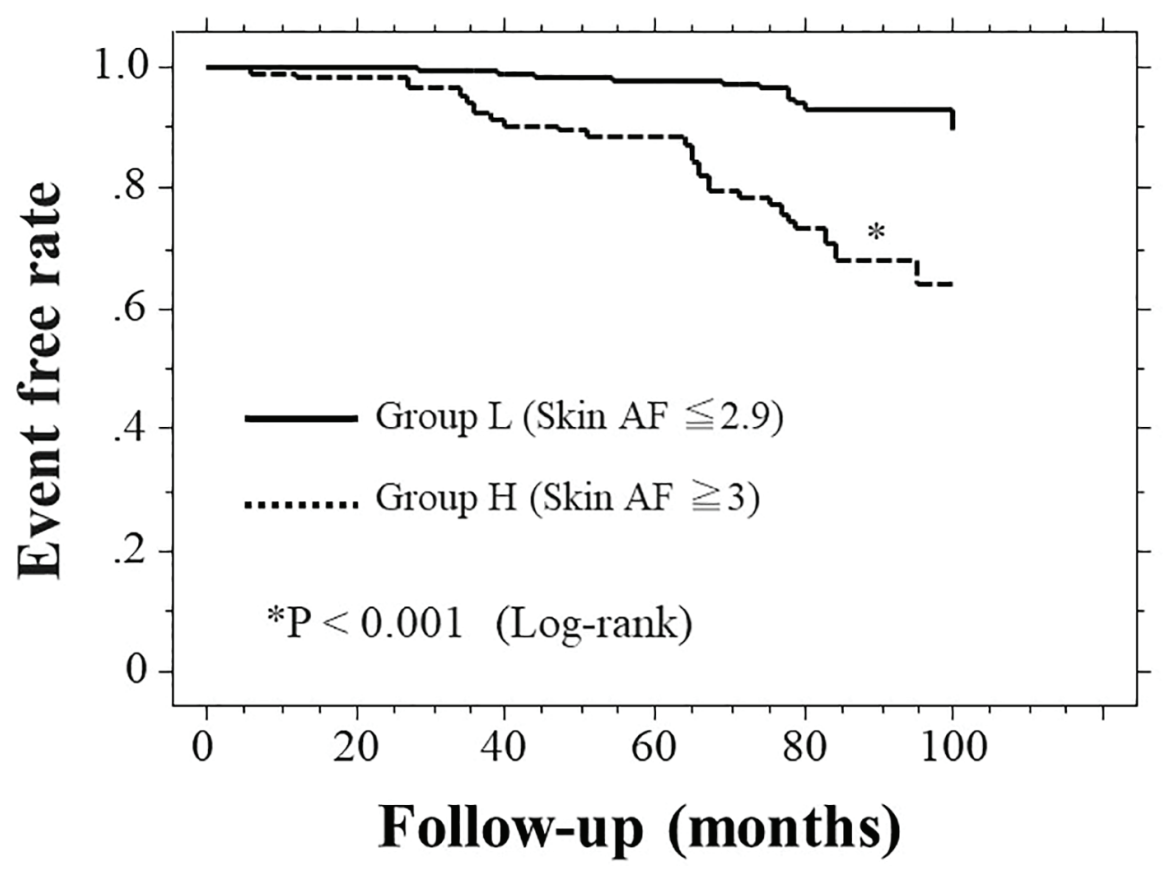

Figure 2. Kaplan-Meier curve for the incidence of first heart failure hospitalization. Patients were followed up for a median of 72.7 months (range, 6 - 100 months). During the follow-up period, $43 \mathrm{HF}$ cases were hospitalized (group L, 15 cases; group H, 28 cases). The Kaplan-Meier curve demonstrated that group $\mathrm{H}$ exhibited a significantly higher incidence of first HF hospitalization than group $L(P<0.001$, log-rank test). HF: heart failure; AF: autofluorescence; AU: arbitrary units.

arterial stiffness or endothelial dysfunction, and CHF prognosis, including HF re-hospitalization $[37,38]$. The results of this study, which indicate a significant association between CAVI values and first HF hospitalization, can be interpreted so that arterial function is an essential factor in the prognosis of patients with HFpEF. However, patients with high skin AF levels showed significantly higher CAVI values than those with low skin AF levels in this study. Basic research studies have reported that AGEs/RAGEs are associated with vascular cell calcification, such as that of endothelial or smooth muscle cells, and functional arterial aging [39-41]. Moreover, clinical studies indicated that skin AF levels significantly correlated with physiological markers of arterial function [10, 42, 43]. Thus, the results of this and previous studies indicate that HFpEF patients with high skin AF levels can be considered a population at high risk of a poor prognosis from the perspective of arterial function.

A number of studies have clarified the importance of inflammation in pathogenesis of CHF. Among the inflammatory markers, hs-CRP levels are commonly used in clinical practice. Moreover, previous studies have reported hs-CRP levels to be independent predictors in CHF patients [44]. The results of this study also indicated that an increase in hs-CRP levels was an independent predictor of first HF hospitalization in patients with HFpEF. However, hs-CRP levels were significantly higher in patients with high skin AF than in those with low skin AF. Researchers have identified a close relationship between AGEs/RAGEs and inflammation in cardiac and vascular cells $[45,46]$. Actually, several clinical studies have reported significant associations between inflammatory markers and skin
AF $[47,48]$. Therefore, the results of the previous and present studies indicate an association of inflammation and AGEs/ RAGEs in the heart or blood vessels, consequently promoting the risk of first HF hospitalization in patients with HFpEF. However, previous studies indicated that both inflammation and AGEs/RAGEs were also associated with pathogenesis of HFrEF $[49,50]$. The present study was not evaluated in patients with HFrEF. Therefore, furher research is required to clarify the relation of skin AF and inflammatory markers in patients with HFrEF.

\section{Limitations}

This study has several limitations. First, this study was conducted at a single center with a relatively small sample size. Thus, the findings cannot be generalized to all medical centers. Second, skin AF was measured only at one time point upon registration. Additional evaluations of correlations between serial changes in skin AF and HF hospitalization are required. Finally, further studies concerning patients with high skin AF are warranted to determine whether aggressive interventional therapy, such as improvements in physical activity or medication, reduces the incidence of first HF hospitalization in patients with $\mathrm{HFpEF}$.

\section{Conclusions}

In conclusion, the present study demonstrated that skin AF 
Table 2. Clinical Parameters at Registration of Patients With and Without Heart Failure Hospitalization

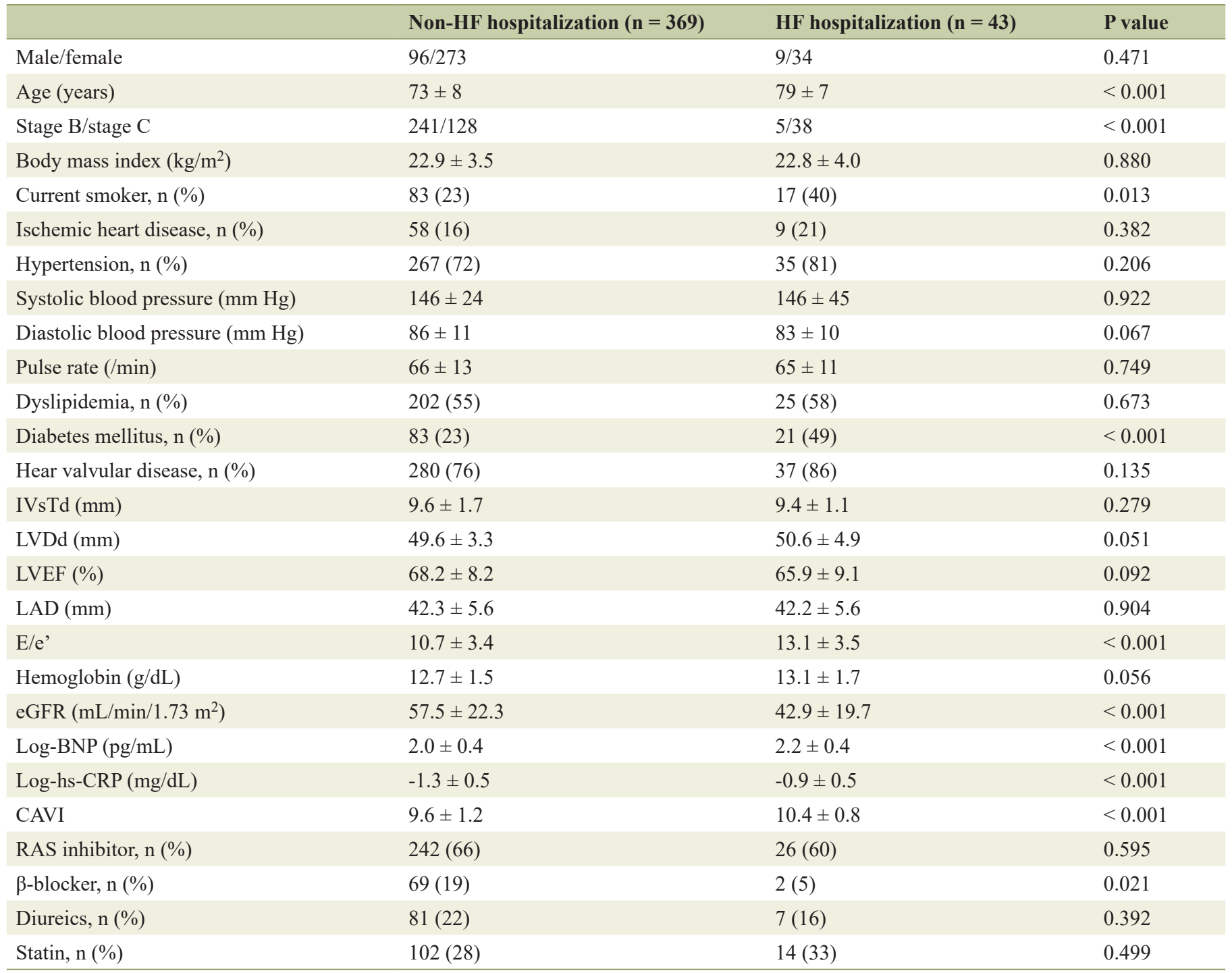

Continuous values are mean \pm SD. HF: heart failure; IVSTd: interventricular septal thickness at end- diastole; LVDd: left ventricular end-diastolic diameter; LVEF: left ventricular ejection fraction; LAD: left atrial dimension; eGFR: estimated glomerular filtration rate; BNP: brain natriuretic peptide; hs-CRP: high-sensitivity C-reactive protein; CAVI: cardio-ankle vascular index; RAS: renin-angiotensin system.

can predict first HF hospitalization in patients with HFpEF. Further prospective studies, including studies investigating intervention therapies, are required to validate the results in this study.

\section{Acknowledgments}

The author is grateful to the individuals who participated in this study.

\section{Financial Disclosure}

None to declare.

\section{Conflict of Interest}

None to declare.

\section{Informed Consent}

All patients provided informed consent.

\section{Author Contributions}

The author was involved in preparing the study design as well as in the acquisition, analysis and interpretation of data. 
Table 3. Multivariate Cox Regression Analysis for Heart Failure Hospitalization

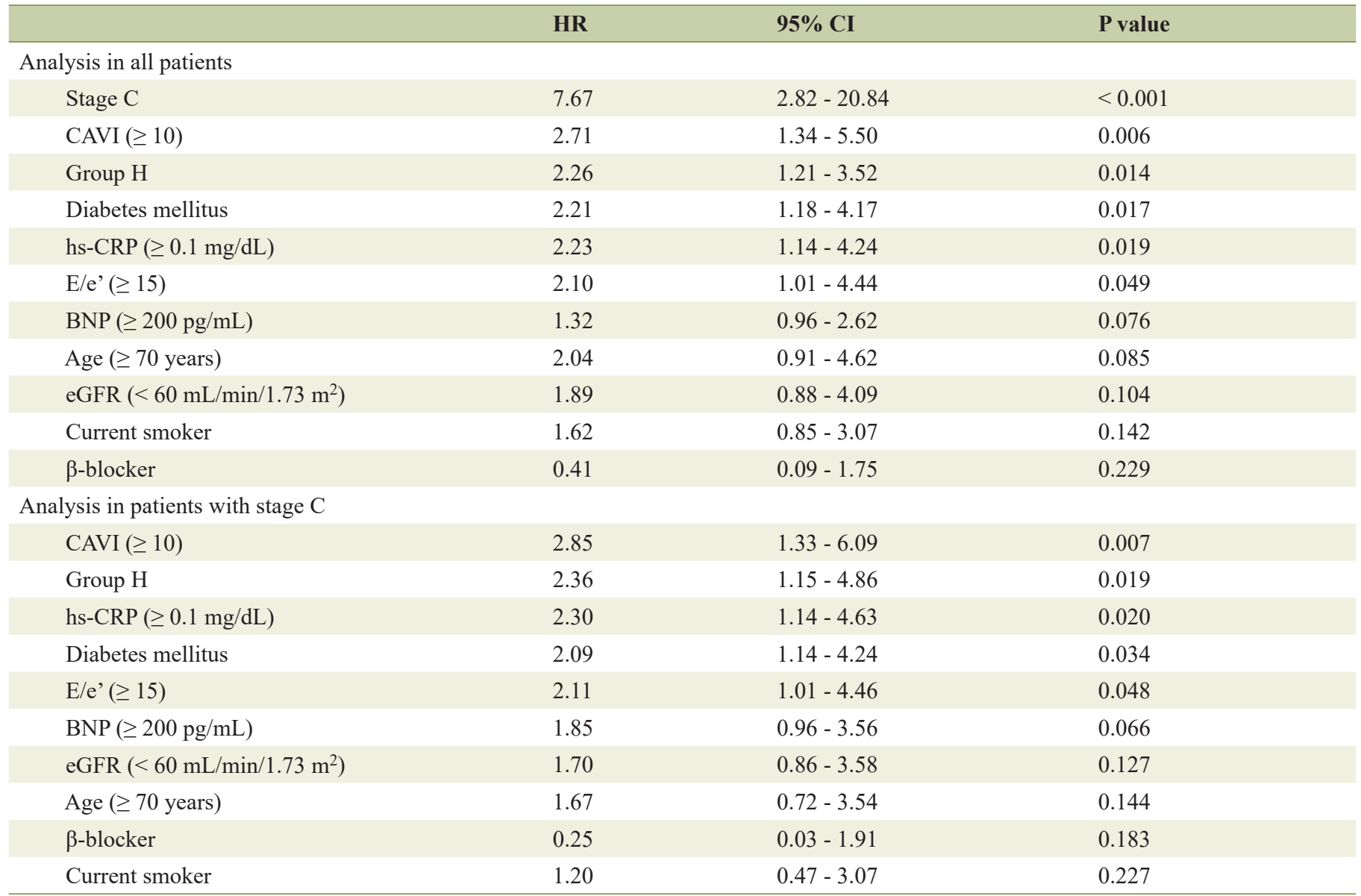

HR: hazard ratio; Cl: confidence interval; CAVI: cardio-ankle vascular index; hs-CRP: high-sensitivity C-reactive protein; BNP: brain natriuretic peptide; eGFR: estimated glomerular filtration rate.

\section{Data Availability}

The author declares that data supporting the findings of this study are available within the article.

\section{References}

1. Haldeman GA, Croft JB, Giles WH, Rashidee A. Hospitalization of patients with heart failure: National Hospital Discharge Survey, 1985 to 1995. Am Heart J. 1999;137(2):352-360.

2. Saczynski JS, Darling CE, Spencer FA, Lessard D, Gore JM, Goldberg RJ. Clinical features, treatment practices, and hospital and long-term outcomes of older patients hospitalized with decompensated heart failure: The Worcester Heart Failure Study. J Am Geriatr Soc. 2009;57(9):1587-1594.

3. Shiba N, Watanabe J, Shinozaki T, Koseki Y, Sakuma M, Kagaya Y, Shirato K, et al. Analysis of chronic heart failure registry in the Tohoku district: third year follow-up. Circ J. 2004;68(5):427-434.
4. Ruigomez A, Michel A, Martin-Perez M, Garcia Rodriguez LA. Heart failure hospitalization: An important prognostic factor for heart failure re-admission and mortality. Int J Cardiol. 2016;220:855-861.

5. Bhatia RS, Tu JV, Lee DS, Austin PC, Fang J, Haouzi A, Gong Y, et al. Outcome of heart failure with preserved ejection fraction in a population-based study. N Engl J Med. 2006;355(3):260-269.

6. Hernandez AF, Hammill BG, O'Connor CM, Schulman KA, Curtis LH, Fonarow GC. Clinical effectiveness of beta-blockers in heart failure: findings from the OPTIMIZE-HF (Organized Program to Initiate Lifesaving Treatment in Hospitalized Patients with Heart Failure) Registry. J Am Coll Cardiol. 2009;53(2):184-192.

7. Tsuchihashi-Makaya M, Hamaguchi S, Kinugawa S, Yokota T, Goto D, Yokoshiki H, Kato N, et al. Characteristics and outcomes of hospitalized patients with heart failure and reduced vs preserved ejection fraction. Report from the Japanese Cardiac Registry of Heart Failure in Cardiology (JCARE-CARD). Circ J. 2009;73(10):18931900.

8. Furuya F, Shimura H, Takahashi K, Akiyama D, Motosugi 
A, Ikegishi Y, Haraguchi K, et al. Skin autofluorescence is a predictor of cardiovascular disease in chronic kidney disease patients. Ther Apher Dial. 2015;19(1):40-44.

9. Noordzij MJ, Mulder DJ, Oomen PH, Brouwer T, Jager J, Castro Cabezas M, Lefrandt JD, et al. Skin autofluorescence and risk of micro- and macrovascular complications in patients with Type 2 diabetes mellitus-a multicentre study. Diabet Med. 2012;29(12):1556-1561.

10. Hitsumoto T. Relationships Between Skin Autofluorescence and Cardio-Ankle Vascular Index in Japanese Male Patients With Metabolic Syndrome. Cardiol Res. 2019;10(3):172-180.

11. Willemsen S, Hartog JW, Hummel YM, van Ruijven MH, van der Horst IC, van Veldhuisen DJ, Voors AA. Tissue advanced glycation end products are associated with diastolic function and aerobic exercise capacity in diabetic heart failure patients. Eur J Heart Fail. 2011;13(1):76-82.

12. Hitsumoto T. Clinical Significance of Skin Autofluorescence in Elderly Patients With Long-Standing Persistent Atrial Fibrillation. Cardiol Res. 2019;10(3):181-187.

13. Yoshioka K. Skin autofluorescence is associated with high-sensitive cardiac troponin $\mathrm{T}$, a circulating cardiac biomarker, in Japanese patients with diabetes: A crosssectional study. Diab Vasc Dis Res. 2018;15(6):559-566.

14. Hunt SA, Abraham WT, Chin MH, Feldman AM, Francis GS, Ganiats TG, Jessup M, et al. 2009 focused update incorporated into the ACC/AHA 2005 Guidelines for the Diagnosis and Management of Heart Failure in Adults: a report of the American College of Cardiology Foundation/American Heart Association Task Force on Practice Guidelines: developed in collaboration with the International Society for Heart and Lung Transplantation. Circulation. 2009;119(14):e391-479.

15. Meerwaldt R, Links TP, Graaff R, Hoogenberg K, Lefrandt JD, Baynes JW, Gans RO, et al. Increased accumulation of skin advanced glycation end-products precedes and correlates with clinical manifestation of diabetic neuropathy. Diabetologia. 2005;48(8):1637-1644.

16. Nomoto K, Yagi M, Arita S, Hamada U, Yonei Y. A survey of fluorescence derived from advanced glycation end products in the skin of Japanese: differences with age and measurement location. Anti Aging Med. 2012;9(5):119124.

17. Meerwaldt R, Graaff R, Oomen PHN, Links TP, Jager JJ, Alderson NL, Thorpe SR, et al. Simple non-invasive assessment of advanced glycation endproduct accumulation. Diabetologia. 2004;47(7):1324-1330.

18. Imai E, Horio $M$, Nitta $\mathrm{K}$, Yamagata $\mathrm{K}$, Iseki $\mathrm{K}$, Hara $\mathrm{S}$, Ura N, et al. Estimation of glomerular filtration rate by the MDRD study equation modified for Japanese patients with chronic kidney disease. Clin Exp Nephrol. 2007;11(1):41-50.

19. Shirai K, Utino J, Otsuka K, Takata M. A novel blood pressure-independent arterial wall stiffness parameter; cardio-ankle vascular index (CAVI). J Atheroscler Thromb. 2006;13(2):101-107.

20. Schisterman EF, Perkins NJ, Liu A, Bondell H. Optimal cut-point and its corresponding Youden Index to discriminate individuals using pooled blood samples. Epidemiol- ogy. 2005;16(1):73-81.

21. Investigators S, Yusuf S, Pitt B, Davis CE, Hood WB, Cohn JN. Effect of enalapril on survival in patients with reduced left ventricular ejection fractions and congestive heart failure. N Engl J Med. 1991;325(5):293-302.

22. Cubbon RM, Adams B, Rajwani A, Mercer BN, Patel PA, Gherardi G, Gale CP, et al. Diabetes mellitus is associated with adverse prognosis in chronic heart failure of ischaemic and non-ischaemic aetiology. Diab Vasc Dis Res. 2013;10(4):330-336.

23. Kannel WB, Hjortland M, Castelli WP. Role of diabetes in congestive heart failure: the Framingham study. Am J Cardiol. 1974;34(1):29-34.

24. Nauta JF, Hummel YM, van der Meer P, Lam CSP, Voors AA, van Melle JP. Correlation with invasive left ventricular filling pressures and prognostic relevance of the echocardiographic diastolic parameters used in the 2016 ESC heart failure guidelines and in the 2016 ASE/EACVI recommendations: a systematic review in patients with heart failure with preserved ejection fraction. Eur J Heart Fail. 2018;20(9):1303-1311.

25. Campbell DJ, Somaratne JB, Jenkins AJ, Prior DL, Yii M, Kenny JF, Newcomb AE, et al. Diastolic dysfunction of aging is independent of myocardial structure but associated with plasma advanced glycation end-product levels. PLoS One. 2012;7(11):e49813.

26. Bayraktar A, Canpolat U, Demiri E, Kunak AU, Ozer N, Aksoyek S, Ovunc K, et al. New insights into the mechanisms of diastolic dysfunction in patients with type 2 diabetes. Scand Cardiovasc J. 2015;49(3):142-148.

27. Wang CC, Wang YC, Wang GJ, Shen MY, Chang YL, Liou SY, Chen HC, et al. Skin autofluorescence is associated with inappropriate left ventricular mass and diastolic dysfunction in subjects at risk for cardiovascular disease. Cardiovasc Diabetol. 2017;16(1):15.

28. Hitsumoto T. Clinical Significance of Skin Autofluorescence in Patients With Type 2 Diabetes Mellitus With Chronic Heart Failure. Cardiol Res. 2018;9(2):83-89.

29. Otaki Y, Arimoto T, Takahashi H, Kadowaki S, Ishigaki D, Narumi T, Honda Y, et al. Prognostic value of myocardial damage markers in patients with chronic heart failure with atrial fibrillation. Intern Med. 2014;53(7):661668.

30. Aimo A, Januzzi JL, Jr., Vergaro G, Ripoli A, Latini R, Masson S, Magnoli M, et al. Prognostic value of high-sensitivity troponin $\mathrm{T}$ in chronic heart failure: an individual patient data meta-analysis. Circulation. 2018;137(3):286297.

31. Ma H, Li SY, Xu P, Babcock SA, Dolence EK, Brownlee M, Li J, et al. Advanced glycation endproduct (AGE) accumulation and AGE receptor (RAGE) up-regulation contribute to the onset of diabetic cardiomyopathy. J Cell Mol Med. 2009;13(8B):1751-1764.

32. Bucciarelli LG, Ananthakrishnan R, Hwang YC, Kaneko M, Song F, Sell DR, Strauch C, et al. RAGE and modulation of ischemic injury in the diabetic myocardium. Diabetes. 2008;57(7):1941-1951.

33. Hofmann B, Jacobs K, Navarrete Santos A, Wienke A, Silber RE, Simm A. Relationship between cardiac tissue 
glycation and skin autofluorescence in patients with coronary artery disease. Diabetes Metab. 2015;41(5):410-415.

34. Hitsumoto T. Clinical impact of skin autofluorescence on high-sensitivity troponin $\mathrm{T}$ in hypertensive patients. Clin Hypertens. 2017;23:19.

35. Endo K, Saiki A, Ohira M, Miyashita Y, Shirai K. Cardioankle vascular index may reflect endothelial function in type 2 diabetes. Int J Clin Pract. 2011;65(11):1200-1201.

36. Kim KJ, Lee BW, Kim HM, Shin JY, Kang ES, Cha BS, Lee EJ, et al. Associations between cardio-ankle vascular index and microvascular complications in type 2 diabetes mellitus patients. J Atheroscler Thromb. 2011;18(4):328336.

37. Meguro T, Nagatomo Y, Nagae A, Seki C, Kondou N, Shibata M, Oda Y. Elevated arterial stiffness evaluated by brachial-ankle pulse wave velocity is deleterious for the prognosis of patients with heart failure. Circ J. 2009;73(4):673-680.

38. Zuchi C, Tritto I, Carluccio E, Mattei C, Cattadori G, Ambrosio G. Role of endothelial dysfunction in heart failure. Heart Fail Rev. 2020;25(1):21-30.

39. Yan SF, Ramasamy R, Schmidt AM. The RAGE axis: a fundamental mechanism signaling danger to the vulnerable vasculature. Circ Res. 2010;106(5):842-853.

40. Grossin N, Auger F, Niquet-Leridon C, Durieux N, Montaigne D, Schmidt AM, Susen S, et al. Dietary CMLenriched protein induces functional arterial aging in a RAGE-dependent manner in mice. Mol Nutr Food Res. 2015;59(5):927-938.

41. Suga T, Iso T, Shimizu T, Tanaka T, Yamagishi S, Takeuchi $\mathrm{M}$, Imaizumi T, et al. Activation of receptor for advanced glycation end products induces osteogenic differentiation of vascular smooth muscle cells. J Atheroscler Thromb. 2011;18(8):670-683.

42. Couppe C, Dall CH, Svensson RB, Olsen RH, Karlsen A, Praet S, Prescott E, et al. Skin autofluorescence is associated with arterial stiffness and insulin level in endurance runners and healthy controls - Effects of aging and endurance exercise. Exp Gerontol. 2017;91:9-14.

43. Wang CC, Wang YC, Wang GJ, Shen MY, Chang YL, Liou SY, Chen HC, et al. Skin autofluorescence is associated with endothelial dysfunction in uremic subjects on hemodialysis. PLoS One. 2016;11(1):e0147771.

44. Anand IS, Latini R, Florea VG, Kuskowski MA, Rector T, Masson S, Signorini S, et al. C-reactive protein in heart failure: prognostic value and the effect of valsartan. Circulation. 2005;112(10):1428-1434.

45. Neviere R, Yu Y, Wang L, Tessier F, Boulanger E. Implication of advanced glycation end products (Ages) and their receptor (Rage) on myocardial contractile and mitochondrial functions. Glycoconj J. 2016;33(4):607-617.

46. Moldogazieva NT, Mokhosoev IM, Mel'nikova TI, Porozov YB, Terentiev AA. Oxidative stress and Advanced Lipoxidation and Glycation End Products (ALEs and AGEs) in aging and age-related diseases. Oxid Med Cell Longev. 2019;2019:3085756.

47. Makulska I, Szczepanska M, Drozdz D, Polak-Jonkisz D, Zwolinska D. Skin autofluorescence as a novel marker of vascular damage in children and adolescents with chronic kidney disease. Pediatr Nephrol. 2015;30(5):811-819.

48. Mulder DJ, van Haelst PL, Graaff R, Gans RO, Zijlstra F, Smit AJ. Skin autofluorescence is elevated in acute myocardial infarction and is associated with the one-year incidence of major adverse cardiac events. Neth Heart J. 2009;17(4):162-168.

49. Santhanakrishnan R, Chong JP, Ng TP, Ling LH, Sim D, Leong KT, Yeo PS, et al. Growth differentiation factor 15, ST2, high-sensitivity troponin T, and N-terminal pro brain natriuretic peptide in heart failure with preserved vs. reduced ejection fraction. Eur J Heart Fail. 2012;14(12):1338-1347.

50. Paulus WJ, Dal Canto E. Distinct myocardial targets for diabetes therapy in heart failure with preserved or reduced ejection fraction. JACC Heart Fail. 2018;6(1):1-7. 